\title{
Prototyping and Evaluation of Display Media using VR for Art Appreciation Education at School
}

\author{
Shoko Usui *, Katsumi Sato ${ }^{\dagger}$, \\ Tatsuya Horita *
}

\begin{abstract}
We examined the use of VR (Virtual Reality) as one solution for improving the display media adopted in art appreciation classes. Art appreciation is gaining greater importance in Japanese education. However, the existing display media, as teaching tools, do not effectively present artworks for classroom study. Existing teaching tools are incapable of accurately depicting the scale and scope of an actual work or providing a stereoscopic view of it from different directions. Many Japanese art teachers have strongly urged the implementation of these two functions, i.e., realsize and multi-direction display. Therefore, we examined technologies to achieve these functions and then proposed a trial presentation medium, VR-SM (Virtual Reality - School Museum), using virtual-reality technology. We conducted an experiment on art appreciation using high school students to compare VR-SM with conventional presentation media. Consequently, the high school students in our study found that VR-SM was a superior medium for displaying artworks at actual size and for appreciating them from many directions. VR-SM thus proved itself to be a promising solution to the problem of constructing dramatic and stimulating display media for presenting artworks.
\end{abstract}

Keywords: Art Appreciation, Display media, Real size, Multidirectional, Virtual reality.

\section{Introduction}

Recently, in the field of education, a learning method that incorporates communication has been emphasized with the spread of the so-called "Society Constructivism." This method seeks to share knowledge through communication and eventually construct it as wisdom that has social or public value [1]. It has been practiced in school education through activities that encourage students to learn together by communicating with one another.

Since the 1980s, many museums and schools have been offering programs with the aim of deepening art appreciation, particularly in the United States [2] [3] [4]. This has led to an intense focus

\footnotetext{
* Graduate School of Information Sciences, Tohoku University, Miyagi, Japan

$\uparrow$ Graduate School of Educational Informatics Research Division, Tohoku University, Miyagi, Japan

* Graduate School of Information Sciences, Tohoku University, Miyagi, Japan
} 
on activities to promote art education, even in Japanese art museums. These efforts include, for example, the development of art appreciation programs and an art appreciation tools in collaboration with schools [5] [6]. In school art classes, appreciation learning has taken on greater importance for attaining a deeper level of appreciation during discussions on art [7].

In Japanese primary and secondary education, curriculum is created based on the educational guidelines developed by the Ministry of Education, Culture, Sports, Science \& Technology (MEXT). Accordingly, Japanese students are required to take a course called "bijutsuka" (art course) from elementary school through high school. Students usually take an art class for about two hours a week. The Japanese "bijutsuka" art course is typically divided into two parts: "expression" and "appreciation." "Expression" focuses on creating artworks such as painting, sculpture, and so on, while "appreciation" fosters the students' ability to appreciate other artists' artworks.

Increasing the class hours for art appreciation has been encouraged by the MEXT guidelines, based on the policy of placing priority on communicative learning and language activities [8].

Many art teachers are requesting the enrichment of presentation equipment and facilities at school to enhance appreciation instruction [9]. In other words, in addition to improving the professionalism of teachers, the improvement of school infrastructure, in particular presentation equipment and facilities, is essential for students to enjoy better opportunities for appreciation learning.

The future requires the development of presentation media suitable for art appreciation classes. For example, conventional presentation media are not capable of presenting a life-size image of an art piece or exhibiting a three-dimensional work from many directions. These functions are in strong demand by many Japanese art teachers [10]. The use of information and communication technologies (ICT) is one method of resolving the current shortcomings of presentation media.

Virtual-reality (VR) technology merits serious consideration as a promising solution for achieving such functions. A key feature of VR is its ability to give a feeling of immersion; therefore, presentation media adopting VR offers the ability for users to feel the actual size of an artwork and to appreciate it from many directions.

In this paper's research, we examine VR technology that can display a work of art at actual size with the ability to appreciate it from different viewpoints. We present a prototype presentation medium and examine its usefulness through experiments in art appreciation learning with high school students.

\section{Related Work}

Photographs of artworks have been widely used in conventional art classes, and differences in the display media that show these photographs may have a profound effect on appreciation learning for students. To clarify these effects, we compared the results obtained in appreciation classes using three different methods: large-sized photographs, hand-held photographs, and digital media [11]. The results showed that students remembered the keywords of artworks better when they used digital media than the other methods. This suggests the importance of using display media having an "interactive function" in order to stir students' interest toward deeper appreciation. 
Some approaches have used photographs displayed by digital media in art appreciation classes, such as Miru-bijyutsu [12], which is commercially available for school education. Other art appreciation classes use special software on tablet PCs. Aside from school learning tools, Google Arts \& Culture [13] is a successful project that enables users to appreciate art galleries by taking a virtual walk through them on the web. Collection Wall [14] is a huge system that interactively displays approximately 4500 artworks simultaneously on a large-sized (12-meter width) touch screen.

However, these approaches are not optimally effective display media for communicative appreciation in school because they generally require large-scale systems. Moreover, they do not display a work of art in its actual size with the ability to appreciate it from different viewpoints.

Recently, VR systems have shown much progress in various fields' products and services. It was in the 1960s that the original prototype of a head-mounted display (HMD) was developed, creating an immersive VR environment through a device worn on the head and covering the user's eyes [15]. HMD systems have been released by several companies over the past several years, at prices ranging from a few tens of thousands yen up to nearly 100,000 yen. However, VR became widely popularized in 2016, a time called "the first year of VR." Currently, reasonably priced VR systems are finding wide application in daily life.

Even at home, we are now able to enjoy immersive games using HMD-based VR. At sightseeing venues, local governments have shown interest in VR; for example, Matsumoto City in mountainous Nagano Prefecture has set up a district called "VR spot" [16] where visitors can enjoy historical sites and other attractions in VR using smartphones while walking around the city. Regarding efforts by museums, a notable example is the "TNM \& TOPPAN Museum Theater" [17], where the collections of the Tokyo National Museum can be appreciated using virtual reality. In this way, VR is becoming a familiar tool for life in Japan.

Many attempts have been made to use VR data as teaching material in Japan. In the field of medical care and nursing, methods using HMD are employed to teach users how to tie cloth bandages for use in emergency response to disasters [18] as well as for teaching material to simulate surgery using VR-generated anatomical charts [19]. In elementary and secondary education, VR-based teaching materials have been developed to teach students the mechanism of the Moon's appearance [20], and such educational VR materials have also been effectively applied to peace studies [21]. From now on, we can expect VR technology to be innovatively implemented in an unforeseeably broad range of teaching materials.

The TNM \& TOPPAN Museum Theater using VR, mentioned above, can be expected to successfully showcase the functions of "real size" and "multiple directions." However, it is difficult to set up a 300-inch screen in a classroom and use it regularly for appreciation clas-ses. In response to this limitation, we decided to explore the trial production of VRbased art appreciation teaching materials of a size that can be practically used in the classroom. 


\section{Examination of Presentation Medium for Viewing Art}

\subsection{Art at actual size and from multidirectional views}

Art appreciation classes should provide exposure to a wide variety of artworks. Some are easy to present at actual size, while others are difficult. For example, with planar works, such as oil paintings and Japanese Ukiyo-e prints, some of those described in many textbooks, such as "The Starry Night" (1889, Vincent van Gogh, New York Museum of Modern Art, $73.7 \mathrm{~cm} \times 92.1 \mathrm{~cm})$ and "Kanagawa Okinawa" (circa 1831, Katsushika Hokusai, Tokyo National Museum, $25.7 \times 37.9$ $\mathrm{cm}$ ) are relatively small, so actual-size presentation is not difficult.

On the other hand, Picasso's "Guernica" (1937, Queen Sofia Art Center) is quite large at $349 \mathrm{~cm}$ $\times 777 \mathrm{~cm}$. In this case, the painting could be split in print or projected onto a large wall, such as a gymnasium. However, these methods are not optimal due to page seams and the lack of vividness. Presenting a work several meters in size remains a challenge.

A three-dimensional artwork cannot be truly appreciated with a simple front-facing photograph. Presenting a solid object so that it can be viewed from many directions is optimal. For a multidirectional view, a method of physically reproducing a solid piece with a 3D printer and presenting a colored copy is one possibility. However, in addition to the problem of copyright, preparation and preservation are difficult for many works. Furthermore, the world's art includes huge works, such as temples, buildings, and public art such as "David" (circa 1504, Michelangelo, Academia Art Museum), whose height exceeds 5 meters. It is almost impossible to reproduce such a piece at actual size with a typical $3 \mathrm{D}$ printer.

\subsection{Application of VR technology}

Virtual reality technology using a head-mounted display (HMD) is one method of displaying art at real size, from small to large, and it can advance the three-dimensional approaches to appreciating stereoscopic works. Because HMD is associated with known biological effects and there are serious health concerns, including asthenopia, use by students should be monitored. However, VR can expand the learning space in schools where space is limited. VR technology may bring about new discoveries and awareness that could not be obtained through previous methods, so we should actively consider its use with presentation media as well.

We sought to produce a presentation medium using VR that could achieve two goals: (1) Full size: Present artworks at their true size and (2) Multidirectional: Allow for appreciation of threedimensional works from many directions. We wanted to verify that there is a benefit to viewing media using VR compared with conventional presentation media.

\section{Implementation of VR-SM}

We propose a presentation medium using VR for art appreciation study, and its prototype is named "VR-SM: Virtual Reality - School Museum." 


\subsection{Determining objects to present}

In our prototype, we do not aim for features that can present many works, as textbooks do, because VR-SM is used to verify our goals of (1) actual-size and (2) multidirectional capabilities. Since it is not necessary to present multiple works, we used one object in this current trial.

Unlike images of two-dimensional art, the 3D data of stereoscopic works (for example, data owned by museums) are not on public display in many cases. In our prototype, we used relatively precise 3D data [22] of the gold mask of Tutankhamun (circa 1300 BC, the Egyptian Museum) and produced it as a trial with VR-SM. This famous image of the golden mask is pictured in many Japanese art textbooks.

\subsection{Implementation environment}

Implementation of VR-SM uses Oculus Rift CV1, an immersive HMD, and the game development engine Unity. The executable file created by Unity operates on a PC, and the resulting image is transmitted to the Oculus Rift CV 1. The overall implementation of the software was done with Unity. It consists of a conventional classroom, desk, chair, work object, and a system that outputs images from a position in the virtual space to the HMD. The system configuration is shown in Table 1, and Figure 1 shows the system image of VR-SM.

Table 1: System Configuration of VR-SM

\begin{tabular}{l|l}
\hline \hline HMD & Oculus Rift CV1 \\
\hline 3D Implementation & Unity 4.5.4 \\
\hline CPU & Core i7 (i7-6700) \\
\hline RAM & 16 GB \\
\hline GPU & GTX 980 Ti \\
\hline Frame rate & $60 \mathrm{fps}$ \\
\hline
\end{tabular}

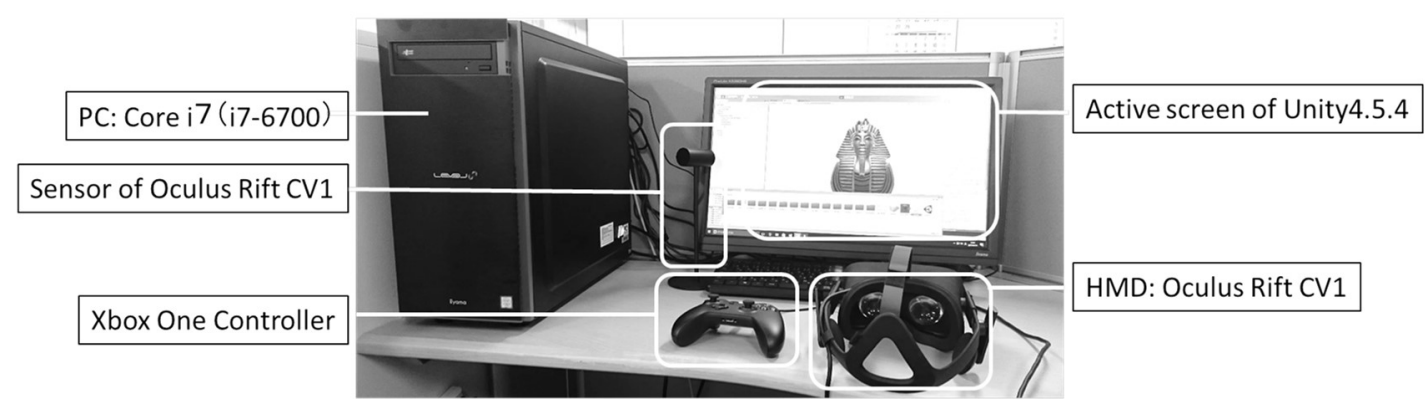

Figure 1: System image of VR-SM 
In order to present the work object at actual size, we set the classroom's desk and chair at a distance that replicates the actual-size perspective on the objects to be compared. The VR-SM uses an Xbox One controller as the basic input device, instead of a keyboard, so that it can interact with the work object. For interaction, we set up controls for zoom up/back, horizontal movement, vertical movement, horizontal rotation, vertical rotation, and rotation by arbitrary rotation axis (Figures 2 and 3).
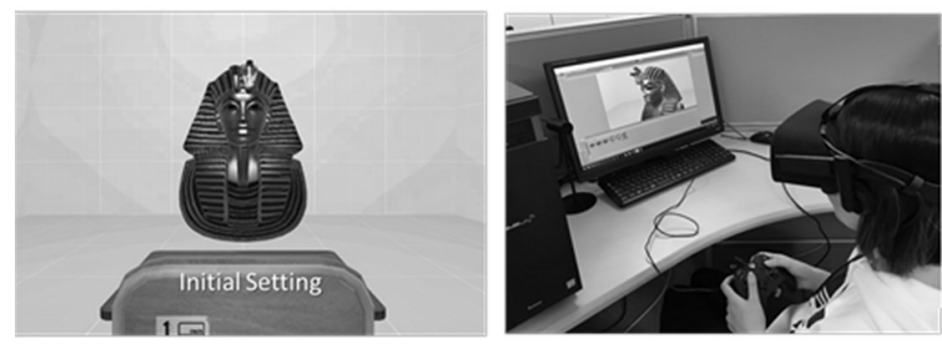

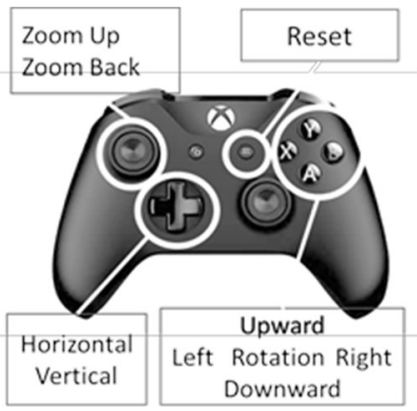

Downward

Figure 2: Scene Using VR-SM with an Interaction Example
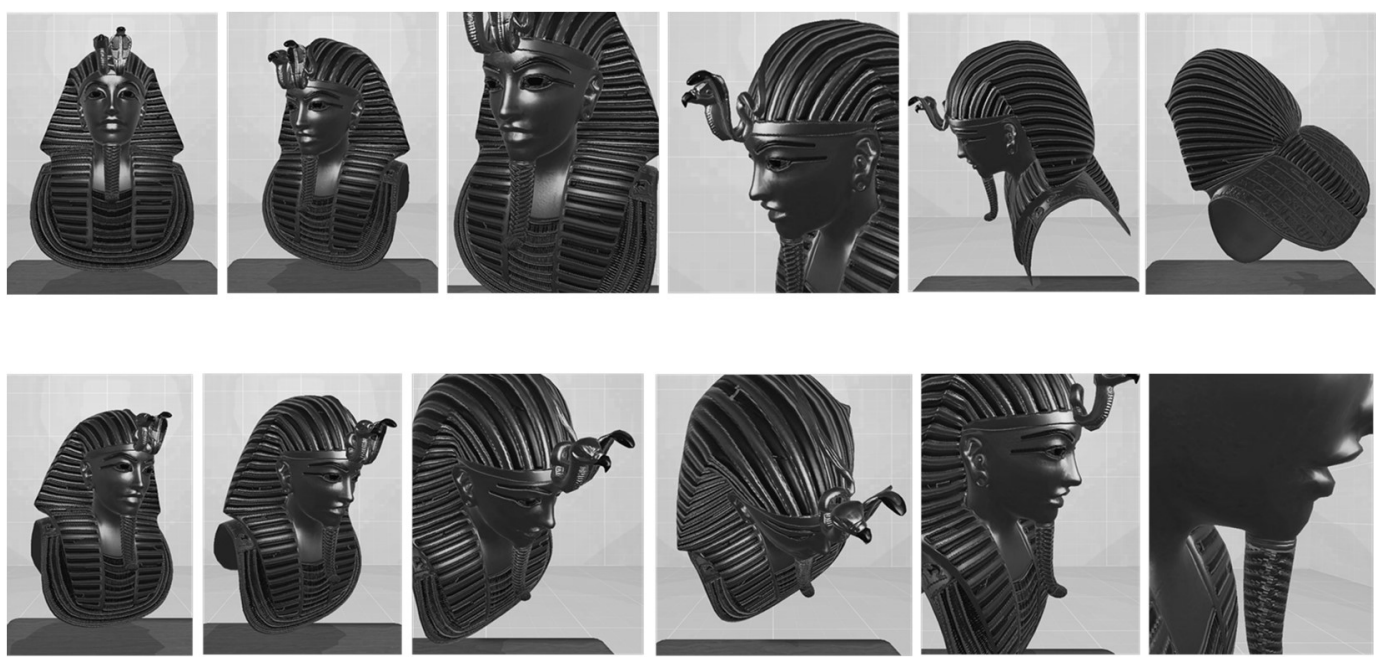

Figure 3: Example of object movement

\subsection{Exploratory Comparison of VR-SM}

In order to confirm the operation of our prototype VR-SM, we carried out exploratory comparisons of the kinds of features the VR-SM has. The initial screen when HMD is installed is shown in Figure 2. Pressing reset on the controller will return it to the initial screen. Figure 4 shows a screen approaching the head of Tutankhamun using the rotation button and zoom-up function. By zooming in, we can confirm that the modeled object at the top of the forehead is reptilian. 
Rotating or moving closer to the head, we note irregularities, such as the letters engraved on the back of the mask (Figure 5). Moreover, there are ear holes (piercings) that appear decorative in nature (Figure 6), and it is possible to clearly confirm that an elongated rod-shaped object is attached under the chin (Figure 7).

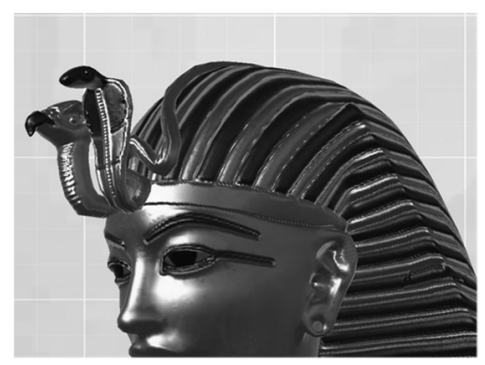

Figure 4: Molding at the top of head

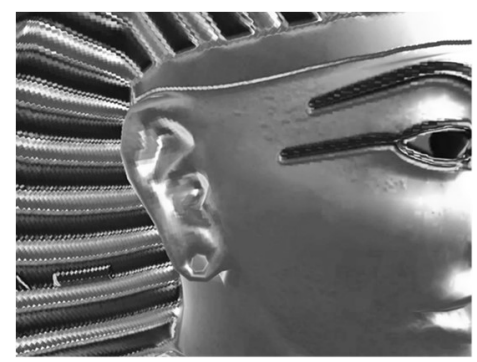

Figure 6: Hole in earlobe

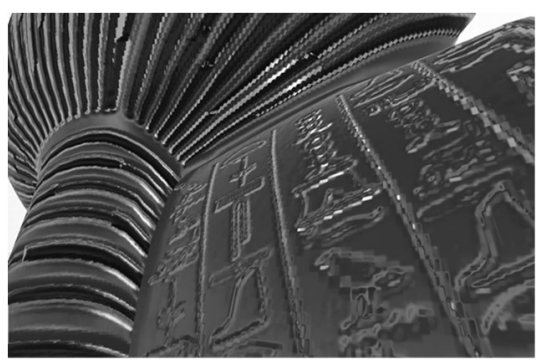

Figure 5: Uneven characters on back

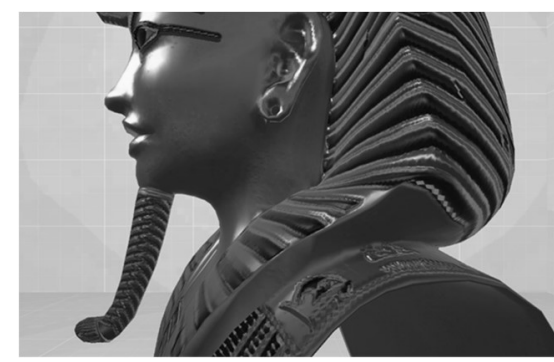

Figure 7: Rod-like beard under chin

A photographic image similar to the golden mask described in the textbook for junior high school students is shown in Figure 8. This textbook provides only small photographs (width $2.5 \times$ height $4.0 \mathrm{~cm}$ ) taken from the front of the piece. Although descriptive information is added to the periphery of the photograph, its small size makes it difficult to perceive the totality and beauty of the modeling, for example, the reptilian object at the top of the mask, the unevenness of the characters engraved on the back, the hole decorations in the ear-lobes, and the rod-shaped beard under the chin. We cannot grasp these details from textbook images.

Using VR-SM enables us to appreciate the finer aspects and shapes of the work that we cannot obtain from photographs used in a class. VR-SM also provides the unique ability to view objects from many directions at their actual size.

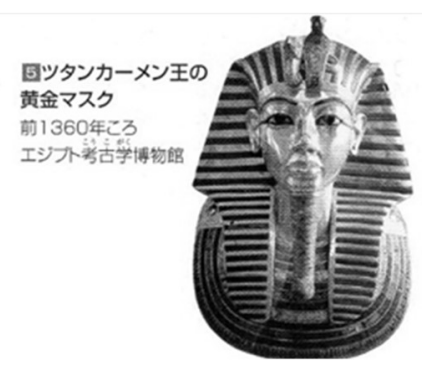

Figure 8: Image of Golden mask in Japanese textbook 


\section{Practical Experiment}

We conducted a comparative practical experiment using VR-SM and existing presentation media. Our motivation was to determine which medium could (1) present at actual size and (2) make it possible to appreciate the piece from multiple directions.

\subsection{Conventional presentation media}

We compared the following four conventional presentation media with our VR-MS.

\section{(A) Textbook}

In Japan, textbooks are the most common type of presentation media used in art classes. In this experiment, we selected photographs taken of the front of the artwork $(2.5 \times 2.5 \mathrm{~cm})$ from textbooks currently on the market.

(B) Poster (actual-size media)

We used a photograph (112 k) obtained by photographing the Tutankhamun mask from approximately the front that was printed on nearly actual-sized photographic paper (width $39.3 \times$ height $54.0 \mathrm{~cm}$ ). This is the only type of media presented at actual size.

(C) Replica (multidirectional media)

A commercially available replica (made of polyresin, width $20.0 \times$ height $30.0 \times$ depth $23.0 \mathrm{~cm}$ ) was prepared as the only medium physically reproducing the solid piece. Its size, however, is smaller than the actual piece.

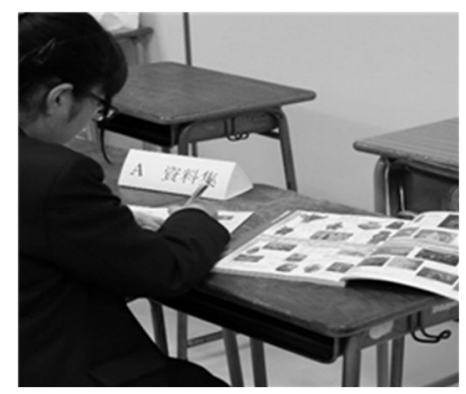

(A) Textbook

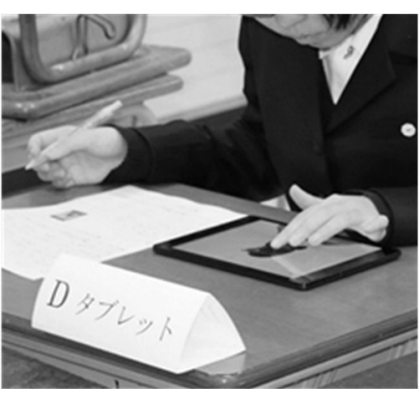

(D) Tablet

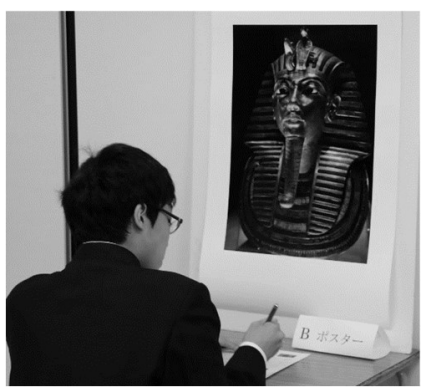

(B) Poster

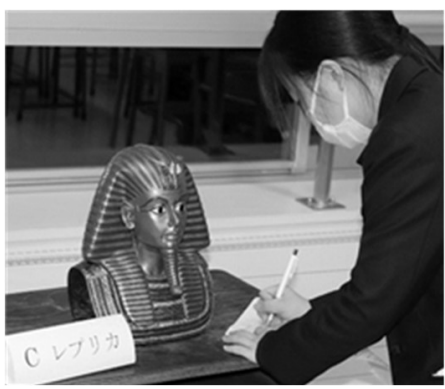

(C) Replica

Figure 9: Conventional Presentation Media and VR-SM 
(D) Tablet

We developed an application that displays the same computer graphics used by VR-SM on a tablet PC. The tablet is equipped with a touchscreen for swipe rotation, up/down and right/left movement, as well as interaction with pinch-in and zoom-out. We also provided a button to reset the position with a tap.

Each presentation medium is shown in Figure 9. In addition, the poster (B) was prepared to present the size of the real thing (actual-size large media) and the replica (C) was prepared to allow viewing from many directions (multidirectional media).

\subsection{Experimental design}

In order to analyze and verify performance in an environment closer to an actual lesson, we conducted an experiment in a school classroom. Considering age restrictions on the use of a VR headset [23], we used high school students as the subjects.

A total of 21 students ( 20 females, 1 male) of N High School participated in the experiment using after-school club activity time (implementation date: November 29, 2016). Participating students received an explanation of the experiment beforehand and agreed to participate after obtaining permission from their parents.

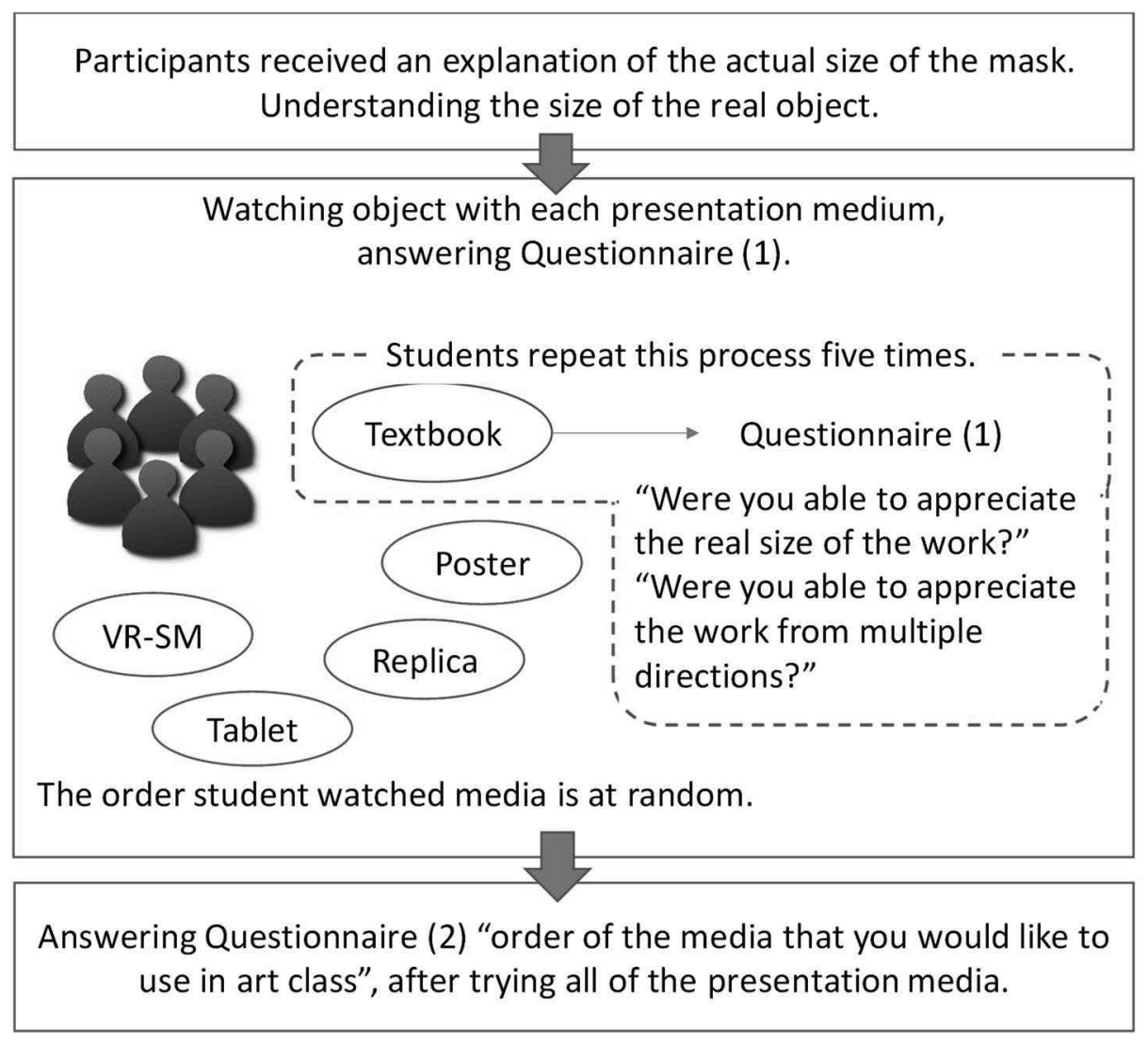

Figure 10: Flow chart of experiment 
After school on the day of the experiment, the participants gathered in an art room and received a simple lecture on the historic background of Tutankhamun's mask. Subsequently, they received an explanation using a paper tape measure (width $39.3 \times$ height $54.0 \times$ depth $49.0 \mathrm{~cm}$ ) to demonstrate the actual size of the mask so that they could understand the size of the real object.

For each presentation medium, students watched it while answering Questionnaire (1). Questionnaire (1) asked, "Were you able to appreciate the real size of the work?" and "Were you able to appreciate the work from multiple directions?" Students answered by selecting one of the following seven choices: 1: I could not appreciate it; 2: intermediate of 1 and 3; 3: I could not appreciate it much; 4 : intermediate of 3 and $5 ; 5$ : I could appreciate it to some extent; 6 : intermediate of 5 and 7; and 7: I was able to appreciate it well.

After trying all of the presentation media, the students answered Questionnaire (2). Questionnaire (2) asked the students to give the "order of the media that you would like to use in art class" and the reasons for their preference.

A practical flowchart of the experiment's procedure is shown in Figure 10. The questionnaires were prepared so as to follow the practice established by Japanese research done to develop and evaluate art teaching materials using AR [24] [25].

The time used for art appreciation was 2 to 5 minutes for each medium, and the time taken by all 21 subjects to try out the five media was about 90 minutes. We observed the activities of the students until the end of the experiment and recorded the art-viewing scenes with a video camera.

\subsection{Experimental results}

Questionnaire (1)

There were no omitted responses, so 21 responses were analyzed for all questions. Table 2 shows the average value and standard deviation (SD) of each of the seven methods. In the actual-size presentation, the average value of VR-SM (5.67) was the highest, followed by the poster (4.57). Furthermore, for multidirectional views, the average value of VR-SM was the highest (6.67), followed by the replica (6.57) and tablet (6.52).

Table 2: Average Value and Standard Deviation of Questionnaire (1)

\begin{tabular}{lcccc}
\hline \hline & \multicolumn{2}{c}{ Real Size } & \multicolumn{2}{c}{ Multidirection } \\
\cline { 2 - 5 } & $\mathrm{M}$ & $S D$ & $\mathrm{M}$ & $S D$ \\
\hline (A) Textbook & 1.29 & 0.72 & 2.95 & 1.28 \\
(B) Poster & 4.57 & 2.11 & 2.62 & 1.94 \\
(C) Replica & 3.81 & 2.14 & 6.57 & 0.81 \\
(D) Tablet & 3.67 & 1.62 & 6.52 & 1.08 \\
\hline (E) VR-SM & 5.67 & 1.32 & 6.67 & 0.97 \\
\hline
\end{tabular}


Rating data obtained by Questionnaire (1) were subjected to two $5 \times 2$ repeated measures analyses of variance (ANOVA) with the factors of media [(A) Textbook, (B) Poster, (C) Replica, (D) Tablet, and (E) VR-MS] and question type [appreciation of artwork's actual size or appreciation of art work from multiple directions]. There were significant main effects of media, $F(4,80)=65.86$, $\mathrm{p}<.001, \eta 2 \mathrm{p}=.76$, and question type, $\mathrm{F}(1,20)=38.93, \mathrm{p}<.001, \eta 2 \mathrm{p}=.66$, as well as an interaction between the two factors, $\mathrm{F}(4,80)=22.28, \mathrm{p}<.001, \eta 2 \mathrm{p}=.52$.

To identify the source of the interaction, we ran simple main effects tests. The simple main effects of media were significant for both question types ("appreciation of size," $F(4,160)-=32.03$, $\mathrm{p}<.001, \eta 2 \mathrm{p}=.61$, and "appreciation from multiple directions," $\mathrm{F}(4,160)=53.52, \mathrm{p}<.001$, $\eta 2 p=.72$ ). Post hoc $t$ tests (Holm-Bonferroni correction for multiple comparisons) revealed that E: VR-SM was valued more positively than A: Textbook, C: Replica, and D: Tablet in the "appreciation of size" condition. In addition, B: Poster, C: Replica and D: Tablet were valued more positively than A: Textbook. On the other hand, in the "appreciation from multiple directions" condition, C: Replica, D: Tablet, and E: VR-SM were valued more positively than A: Textbook and B: Poster.

The poster was printed at full size, so these results likely indicate that VR-SM can present actual size in a way similar to that of a true-to-size poster. Furthermore, since the replicas were smaller than the real artwork, few students responded that they were able to feel the full size. These responses suggest that if the size is different from the actual size, it may be difficult for viewers to imagine the original size.

The replica used was a three-dimensional medium that physically reproduces a solid piece only through conventional media. In other words, the result that C: Replica, D: Tablet, and E: VR-SM were valued more positively than A: Textbook and B: Poster in the "appreciation from multiple directions" condition seems to indicate that Tablet and VR-SM are presentation media that provide multidirectional views similarly to a three-dimensional replica.

Students' ratings of posters and textbooks were low, suggesting that printed material could not effectively elicit appreciation of solid objects. Unlike printed material, VR-SM and tablets can be viewed by moving the artwork on the display many times, and this was inferred as the reason that many students were able to appreciate artworks from many directions. Moreover, it can be conjectured that a large number of students perceived an artwork's actual size from VR-SM than from the poster because they were able to freely view the real object from any direction using VR-SM. These findings suggest the potential of VR-SM as an effective medium for reflecting actual size and for appreciating an artwork from many directions.

Questionnaire (2)

For the question "Presentation medium I would most like to use in art class," most students an-swered that they wanted to use VR-SM (Table 3). We conducted a Friedman test to evaluate the differences by medium for the rank scores. This test was significant $(\chi 2(5)=66.54, p<.001)$. Next, follow-up pair-wise comparisons were conducted using Wilcoxon signed-rank tests with Holm-Bonferroni correction. The results indicate that Replica, Tablet and VR-SM were preferable to Textbook and Poster; in addition, Poster was preferable to Textbook. 
Table 3: Order of Preference for Use of Presentation Media

in Art Appreciation Class by Subject

\begin{tabular}{|c|c|c|c|c|c|c|c|c|c|c|c|c|c|c|c|c|c|c|c|c|c|}
\hline \multirow[t]{2}{*}{ Order } & \multicolumn{21}{|c|}{ Subject number } \\
\hline & 1 & 2 & 3 & 4 & 5 & 6 & 7 & 8 & 9 & 10 & 11 & 12 & 13 & 14 & 15 & 16 & 17 & 18 & 19 & 20 & 21 \\
\hline $1 s t$ & $E$ & E & $E$ & E & $E$ & E & $E$ & E & E & $E$ & $E$ & $E$ & D & D & D & $\mathrm{C}$ & C & C & D & C & C \\
\hline 2nd & D & D & D & D & D & D & D & C & C & D & $\mathrm{C}$ & C & E & E & E & $E$ & $\mathrm{D}$ & D & B & D & B \\
\hline 3rd & C & C & C & B & C & C & C & D & D & C & $\mathrm{D}$ & B & C & C & B & $\mathrm{D}$ & $E$ & $E$ & $E$ & B & A \\
\hline 4th & B & B & B & C & B & B & B & B & B & B & B & D & B & B & C & B & B & B & C & A & D \\
\hline 5 th & A & A & A & A & A & A & A & A & A & A & A & A & A & A & A & A & A & A & A & $E$ & $\mathrm{E}$ \\
\hline
\end{tabular}

Most students answered that they wanted to use VR-SM for reasons such as "It is good to be able to see the details yourself" and that it was "Fun" and "Motivating." MEXT guidelines stipulate that art appreciation provide pleasure and joy to students [8]. VR-SM can thus be considered an effective medium for making the study of art appreciation pleasurable for students.

Many students also wanted to use the Replica and the Tablet in addition to VR-SM. The reasons for choosing Replica included "I do not want to use VR-SM or the tablet at school because it seems like I'm more involved in the pleasure of using the machine itself than in the class contents" and "It is good to be able to see entire images of objects in large numbers."

The reasons for choosing Tablet included "I could enjoy exchanging opinions with my friends" and "I was able to see the object with several other people at the same time." In school classes, it is vitally important for multiple people to be able to see the same image together at the same time for such activities as group work and discussions.

The evaluations of VR-SM by subjects 20 and 21 are low, citing as reasons "Eyes get tired, I get dizzy" and "It is troublesome to remove my glasses." Consequently, in the development of presentation media making use of VR, issues such as reducing health concerns must be clarified.

\section{Discussion}

Conducting research on presentation media for art appreciation is critically important for enabling art teachers to provide better appreciation classes. ICT has expanded the possibilities of presentation media, deploying such powerful functions as expanding the size of an artwork, comparing multiple artworks on a single display, and searching for artworks and related information on the web. However, the key functions of "presenting artwork at actual size" and "ability to appreciate artworks from multiple directions" have not been fully realized in current educational media.

Therefore, in this research, we proposed a viewing medium that can present the actual size of an artwork and display a solid piece from many directions. We examined its usefulness through an experiment with high school students. The presentation medium VR-SM, which was prototyped 
using VR technology, made it possible for students to feel the "real size" of the art they were studying to a greater extent than when using conventional presentation media such as textbooks, replicas and tablets. Furthermore, the students felt VR-SM was useful for appreciating an artwork from multiple directions. From judging our overall results, VR-SM can be considered highly useful for art appreciation classes in school. VR-SM made it possible to use methods that were nearly impossible with conventional presentation media. Moreover, VR-SM is expected to powerfully elicit the interest of students in classes and to lead them to learning more subjectively.

However, we must alert readers to one particular condition in this experiment. The actual school where we conducted the experiments was previously an all-girl high school. Consequently, even now approximately $90 \%$ of the students in the high school's Art Department activities are female, and almost all of the students who participated in this experiment were female. Perhaps slightly different results would be obtained in the case of using male participants, and a comparison study to clarify gender (and other) differences would be an interesting research topic. Therefore, we plan to carry out a quantitative analysis with a greater variety of students in the future.

As another significant consideration, some students showed a weak tendency to use VR-SM from the responses obtained by Questionnaire (2). Their reasons can be summarized as two points:

(1) Consciousness of using ICT equipment overwhelmed learning the contents of the lesson;

(2) Group learning was found to be impossible.

We are giving serious consideration to both of these points. In Japan, there is currently discussion on a proposed policy to provide and maintain a tablet device for each student as well as to enhance various ICT equipment in classrooms [26]; this approach will surely advance the development of an ICT environment in the classroom. Therefore, we believe point (1) is a transient issue due to the large gap between ICT and existing teaching materials. In other words, this problem will likely be resolved by the increasingly positive attitudes of teachers toward changes in the learning environment [27] [28]. Nevertheless, teachers must show ingenuity in helping students to concentrate on the contents of a lesson while using ICT equipment.

Regarding point (2), the current version of VR-SM cannot be used by more than one person at a time. However, this capability is becoming technically possible. We need to develop such future technical improvements in VR-SM to make it a stronger real-world educational tool.

Furthermore, we need to consider a few other issues related to using ICT in education, including the incorporation of VR in teaching materials. One is to improve the skills of teachers in effective ICT operation. Another issue is to improve the ease of use of the ICT equipment itself. More generally, it will be necessary to improve the overall ICT environment of the classroom [29] [30]. In deploying ICT, it will be necessary to resolve such fundamental issues in the future.

In future studies on the use of VR as teaching materials, we will face new challenges regarding the relation between presentation media and education in art appreciation. For example, we plan to clarify what kinds of lessons are possible and what kinds of learning effects can be expected by using presentation media like VR-SM. We must particularly address those functions that working art teachers are eagerly awaiting, such as "seeing artwork at real size" and "being able to appreciate a three-dimensional image from various directions."

Unlike conventional presentation media, the ability of VR-SM to show artworks at real size and 
from multiple directions can be expected to bring new adventures in learning to students. For example, Michelangelo's iconic David is 5 meters high, but the shape of the face as seen from the ground and that seen from David's eye level are quite different. Using the various viewpoints of VR-SM may help to teach students the thought and aim of Michelangelo. In other words, there is the possibility of this medium bringing new discoveries.

We are considering a variety of possibilities for display media using VR in art appreciation classes. Buildings such as castles and temples are also artworks, and these structures are also described in textbooks for art classes in Japan. In the case of such a huge work or a fixed sculpture, it is natural for the viewer to walk around and take in different viewpoints rather than moving the artwork itself in the VR space. In other words, we need a VR system that allows viewers themselves to move and thus virtually walk around the artwork. Prototyping a system that lets users move around a few meters while wearing HMD is technically possible. In addition, the development of technology that allows computers to be built into HMDs and used wirelessly is also progressing [31]. When wireless HMD is used at school, further issues such as safety considerations are expected.

The development of VR technology is still being innovated, and various attempts are expected for application to teaching materials. In this research, we examined the use of VR as teaching materials for art appreciation and utilized VR for display media. As we mentioned in the related work of previous studies, efforts to utilize VR as teaching material are increasing in Japan. However, research on using VR in art classes has only just begun, so we believe our own research is important within the framework of this growing educational trend.

In Japan, there is often no museum located near a school. In the future, in addition to addressing the issue of health risks from the use of VR-SM, we would like to consider installing permanent VR museums in schools to allow students to appreciate art from around the world.

\section{Acknowledgments}

We appreciate the contributions of the students and faculty members who cooperated in the experiment. This work is supported by the 11th Hakuho Research Grant for Child Education, Hakuho Foundation.

\section{Author's Note}

This thesis was modified by adding new information to "Development and Evaluation Experiment of Display Media using VR for Art Appreciation Learning," which we presented at the IIAI International Congress on Advanced Applied Informatics, IIAI AAI 2017.

\section{References}

[1] Gergen, K.J., "Toward Transformation in Social Knowledge," 2nd ed., London, Sage Publications, 1994. 
[2] The Museum of Modern Art New York, "The Museum of Modern Art New York Annul Report 1984-85," p. 42, 1985.

[3] Arenas, A., "Is this Art?" Art Education Association, Virginia, vol. 43, no. 5, pp. 25-28, 4549, 1990.

[4] The Getty Center for Education in the Arts. Discipline-Based Art Education, Curriculum Sampler, The Getty Center, USA, 1991.

[5] Arenas, A., Kawamura Memorial DIC Museum of Art, "Is this Art?" Tankosha Publishing Co., Ltd., p. 199, 1998.

[6] Yamaki, A., et al., "Art Appreciation declaration school + art museum," Nihon Bunkyou Shuppan Co., Ltd., p. 355, 2003.

[7] Ueno, K., "Why are Fujin Raijin laughing?" Appreciation by dialogue complete guide, Mitsumura Tosho Publishing Co., Ltd., p. 295, 2014.

[8] Ministry of Education, Culture, Sports, Science and Technology (MEXT), "Curriculum Guideline Commentary of Junior High School for Art," 2008.

[9] Art Education Society of Japan Research Division, "The investigation report for Appreciation teaching in School of Art Department - 2015 national survey results," http://www.aesj.org/ (accessed 2017. 09. 20).

[10] Usui, S., Sato, K., "A Study of Survey for Implementation of Art Appreciation Media," The 39th Annual Conference, The Association of Art Education, Japan, p. 018, 2017.

[11] Usui, S., Yokoyama, H., Kitamura, Y., "A Study of Impacts of Showing Media on Art Appreciation," The Journal for Japan Society for Educational Technology, 38, Suppl., pp. 45-48, 2014.

[12] Miru-bijyutsu. Nihon Bunkyou Shuppan Co., Ltd., https://www.nichibun-g.co.jp/textbooks/digital/mirubi/ (accessed 2017. 09. 20).

[13] Google. Google Art \& Culture, https://www.google.com/culturalinstitute/project/artproject (accessed 2017. 09. 20).

[14] Collection Wall. The Cleveland Museum of Art. http://www.clevelandart.org/galleryone/collection-wall/ (accessed 2017. 09. 20).

[15] Tachi, S., Sato, M., Hirose, M., "Virtual Reality Studies." CORONA PUBLISHING CO., LTD., 2011.

[16] Matsumoto City, Nagano Prefecture, https://www.city.matsumoto.nagano.jp/miryoku/siro/osirase/matsumotocastleVR.html (accessed 2017.09.20).

[17] Tokyo National Museum, TOPPAN PRINTING CO., LTD., “TNM \& TOPPAN Museum Theater." http://www.toppan-VR.jp/mt/about/ (accessed 2017.09.20).

[18] Shibata, T., Harada, M., "Educational Tool for First Aid Training Using an Optical See- 
through Head Mounted Display.” The Journal for Educational Media Vol. 23(2), pp. 35-45, 2017.

[19] Sugimoto, M., “VR/AR Medical shock.” Born Digital, Inc., 2017.

[20] Setozaki, N., Morita, Y., Takeda, T., "Development of Multi Aspect Type VR Teaching Material Based on Needs Investigation and Practice Class." Transactions of the Virtual Reality Society of Japan, Vol. 11(4), pp. 537-543, 2006.

[21] Setozaki, N., Sato, K., "Examination of the Effective Use of Spherical Panorama VR Learning Material in a Practice Class of Peace Education." The Journal for Educational Media Vol. 23(2), pp. 15-24, 2017.

[22] TurboSquid, "3D MODELS FOR PROFESSIONALS," https://www.turbosquid.com/3dmodels/pharaon-mask-3d-model/232649/ (accessed 2017. 09. 20).

[23] "Oculus Lift, Regal Documents, Health and Safety Warnings," https://www.oculus.com/legal/health-and-safety-warnings/ (accessed 2017. 09. 20).

[24] Setozaki, N., Kato, T., Terashi, W., Iwasaki, T., Morita, Y., "Development and Evaluation of Art Teaching Equipment Using AR technology," IEICE technical report, 110(405), pp. 23-26, 2011.

[25] Setozaki, N., Kato, T., Terashi, W., Iwasaki, T., Morita, Y., "Examination about Utility of Active Manipulation using AR Teaching Equipment for Statue Appreciation," The Journal for Japan Society for Educational Technology, 35(Suppl.), pp. 105-108, 2011.

[26] Ministry of Education, Culture, Sports, Science and Technology (MEXT), "Expert Opinion Meeting on the Ideal Way to Improve ICT Environment at School," Final summary, http://www.mext.go.jp/component/b_menu/shingi/toushin/_icsFiles/afieldfile/2017/12/13/1388920_1.pdf, 2017, (accessed 2017. 09. 20).

[27] Hooper, S., \& Rieber, L. P. “Teaching with Technology,” In A. C. Ornstein (Ed.), Teaching: Theory into practice, Needham Heights, MA: Ally and Bacon, pp. 154-170, 1995.

[28] UNESCO. "ICT Transforming Education A Regional Gide," http://unesdoc.unesco.org/images/0018/001892/189216e.pdf, (accessed 2017. 09. 20).

[29] Kondo, I., Kurokami, H., Horita, T., Nonaka, Y., Development and utilization of educational media, Minerva Shobo, 2015, p. 190.

[30] Venezky, R. L., Davis, C., "Quo Vademus? The Transformation of Schooling in a Networked World," OECD / CERI, http://fatih.inetd.org.tr/oecd-2002-ict-school.pdf, (accessed 2017. 09. 20).

[31] Taiwan HTC, Vive Focus, https://www.vive.com/cn/product/vive-focus-en/, (accessed 2017. 09. 20). 